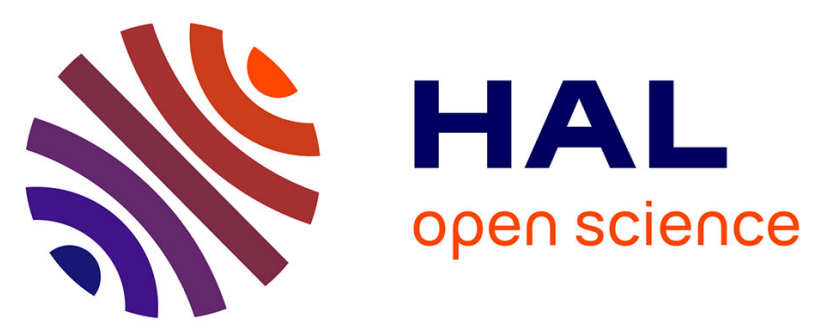

\title{
Unsupervised learning of seismic wavefield features: clustering continuous array seismic data during the 2009 L'Aquila earthquake
}

Peidong Shi, Léonard Seydoux, Piero Poli

\section{- To cite this version:}

Peidong Shi, Léonard Seydoux, Piero Poli. Unsupervised learning of seismic wavefield features: clustering continuous array seismic data during the 2009 L'Aquila earthquake. Journal of Geophysical Research: Solid Earth, 2020, 10.1029/2020JB020506 . insu-03094514

HAL Id: insu-03094514

https://hal-insu.archives-ouvertes.fr/insu-03094514

Submitted on 4 Jan 2021

HAL is a multi-disciplinary open access archive for the deposit and dissemination of scientific research documents, whether they are published or not. The documents may come from teaching and research institutions in France or abroad, or from public or private research centers.
L'archive ouverte pluridisciplinaire HAL, est destinée au dépôt et à la diffusion de documents scientifiques de niveau recherche, publiés ou non, émanant des établissements d'enseignement et de recherche français ou étrangers, des laboratoires publics ou privés. 


\title{
Unsupervised learning of seismic wavefield features: clustering continuous array seismic data during the 2009 L'Aquila earthquake
}

\author{
Peidong Shi ${ }^{1}$, Léonard Seydoux ${ }^{1}$, and Piero Poli ${ }^{1}$ \\ ${ }^{1}$ Institut de Sciences de la Terre, Université Grenoble Alpes, CNRS (UMR5275), Grenoble, France.
}

Key Points:

- Identification of frequency-dependent wavefield features from array analysis of continuous seismic data

- Wavefield features reveal a time and frequency evolution of seismic wavefield related to the seismic source properties and fault states

- Unsupervised learning of wavefield features identifies distinct clusters well correlated with different periods of seismic cycle without explicit physical modeling

Corresponding author: Peidong Shi, peidong.shi@univ-grenoble-alpes.fr 


\begin{abstract}
We apply unsupervised machine learning to three years of continuous seismic data to unravel the evolution of seismic wavefield properties in the period of the 2009 L'Aquila earthquake. To obtain sensible representations of the wavefield properties variations, we extract wavefield features (i.e. entropy, coherency, eigenvalue variance and first eigenvalue) from the covariance matrix analysis of the continuous wavefield data. The defined wavefield features are insensitive to site-dependent local noise, and inform the spatiotemporal properties of seismic waves generated by sources inside the array. We perform a sensitivity analysis of these wavefield features, and track the evolution of source properties from the unsupervised learning of the uncorrelated features. By clustering the wavefield features, our unsupervised analysis avoids explicit physical modeling (e.g. no requirement for event location and magnitude estimation) and can naturally separate peculiar patterns solely from continuous seismic data. Our model-free unsupervised learning of wavefield features reveals distinct clusters well correlated with different periods of the seismic cycle, which are consistent with previous model-dependent studies.
\end{abstract}

\title{
1 Introduction
}

Seismological observations are a primary source of information about fault physics and its evolution in time and space (Gutenberg \& Richter, 1956; Scholz, 2002; Aki \& Richards, 2002). Seismic catalogs are nowadays the main way of labeling seismic data, by association of waveforms with earthquakes occurring in a given position and at a certain time (Gutenberg \& Richter, 1956; Scholz, 2002; Aki \& Richards, 2002). While earthquake catalogs are among the main source of information to study faults, the continuous stream of seismic data is likely to hide important additional information about fault physics, which cannot be easily summarized into discrete observables. For example, the slow earthquakes and tremors show very different wavefield properties compared to that of regular earthquakes, requiring alternative approaches to derive information about their physics (Ide et al., 2007; Beroza \& Ide, 2011). Therefore, it is worthwhile to explore the potential to assess physical properties of faults from direct analysis of continuous seismic wavefields.

The latter idea has been recently explored in laboratory-scale fracture experiments. Indeed, recent studies based on laboratory observations, show that continuous acoustic emission (AE) contain essential information about the physical state of the rock (RouetLeduc et al., 2017; Bolton et al., 2019; Hulbert et al., 2019). In these studies, statistical features of the continuous AE signals (e.g. amplitudes, variance etc.), are used for supervised or unsupervised machine learning (ML) and classification, to characterize the wavefield variations and study the evolution of the (laboratory) seismic cycle (Bolton et al., 2019), including the estimation of failure time (Rouet-Leduc et al., 2017). The experiments carried at a laboratory scale already involve complex, nonlinear relationships between the continuous signal properties and the fault states, suggesting that systems of higher complexity such as the real geological settings should also be investigated with machine learning tools, as in the present study.

In addition to the laboratory studies, unsupervised machine learning has been applied to real continuous seismic data in volcanic settings to classify volcanic tremors and monitor volcanic activities (Langer et al., 2009; Esposito et al., 2008; Köhler et al., 2010; Langer et al., 2011; Carniel et al., 2013; Unglert et al., 2016). Unsupervised machine learning can distinguish seismic wavefield of distinct characteristics (e.g. spectral content) generated by different volcanic activities, such as pre-, co- and post-eruption, thus permits the recognition of different types of volcanic activities directly from continuous seismic data.

In summary, both laboratory experiments (Rouet-Leduc et al., 2017; Bolton et al., 2019; Hulbert et al., 2019; Shreedharan et al., 2020) and real volcanic seismic data analysis (Esposito et al., 2008; Köhler et al., 2010; Langer et al., 2011; Carniel et al., 2013; 
Unglert et al., 2016) show promising potential to utilize real continuous seismic wavefield and ML algorithms to understand physical processes occurring inside the Earth. However, to our knowledge, no studies have been performed so far on clustering of long-term real continuous array seismic data to establish the space-time evolution of the physical state of the faults where significant earthquakes occur.

We here present an unsupervised class-membership identification (clustering) of ensemble wavefield features, which capture the nature of the seismic wavefield as seen by an array of stations. The choice of array features is aimed at reducing the sensitivity of single-station statistical features to noise intensity (e.g. daily/weekly variation of human activity and variation of meteorological conditions, Cara et al., 2003; Poli et al., 2020) and enhancing the identification of spatio-temporal properties of (possibly mixed) seismic sources (Seydoux et al., 2016a; Soubestre et al., 2019). We can thus recognize patterns within seismic signals and track their temporal evolution, which can be related to particular fault states occurring at different stages of the seismic cycle (e.g. earthquake nucleation, afterslip etc.). Differently from laboratory experiments (Rouet-Leduc et al., 2017; Hulbert et al., 2019; Shreedharan et al., 2020), we have no independent information about the fault state (e.g. stress, friction). That is why we use unsupervised analysis and self-learn from the continuous data.

To test our approach, we used three years of vertical-component seismic data recorded in the region of L'Aquila, Italy (Figure 1). We use this region as a test case, as it hosting a magnitude 6 earthquake ( 6 of April, 2009, Chiarabba et al., 2009; Di Luccio et al., 2010) preceded by a long-lasting preparatory phase (Sugan et al., 2014; Vuan et al., 2018). Previous studies also reported that the fault properties may have changed dramatically in the preparatory phase of the main event due to fluid movement (Di Luccio et al., 2010; Chiarabba et al., 2020), velocity change (Baccheschi et al., 2020), and variation of elastic and anisotropic parameters (Lucente et al., 2010). In addition, this region is well instrumented with permanent seismic stations (Figure 1a), allowing an array-based analysis. The complex faulting processes and high quality continuous seismic data make the L'Aquila earthquake a perfect test case to investigate the feasibility of tracking fault states directly from continuous seismic wavefield.

We explore spatial wavefield features of long time windows (60 days) and their temporal evolution with respect to the main earthquake in the area using cluster analysis. We highlight different patterns in the wavefield and relate them to the physical processes of the fault (e.g. the preparation, afterslip etc.). Our results show the feasibility of using array-based wavefield properties to directly assess the fault state and characterize different stages of the seismic cycle.

\section{Data and Processing}

We focus on a time period of about 3 years (2008-2010, included) around the Mw 6.1 L'Aquila earthquake (6 April 2009, Chiarabba et al., 2009; Di Luccio et al., 2010). This event has been chosen because it presented a prominent and long-lasting preparation period, starting 3-4 months before the mainshock, and including several dozens of foreshocks and possible significant changes in the fault rock properties (Di Luccio et al., 2010; Lucente et al., 2010; Di Stefano et al., 2011; Herrmann et al., 2011; Sugan et al., 2014; Vuan et al., 2018; Chiarabba et al., 2020).

The three years of continuous vertical-component seismic data (from 2007-11-03 to 2010-08-23) recorded by the six nearest stations (Figure 1a) at a $50 \mathrm{~Hz}$ sampling rate were downloaded from the Istituto Nazionale Geofisica e Vulcanologia (INGV) data center (INGV Seismological Data Centre, 2006). Data have been transformed into velocity using the instrument response and processed to remove gaps and glitches. Data gaps and glitches are filled or replaced with white random noise of minimal amplitudes $(\sim 10$ 

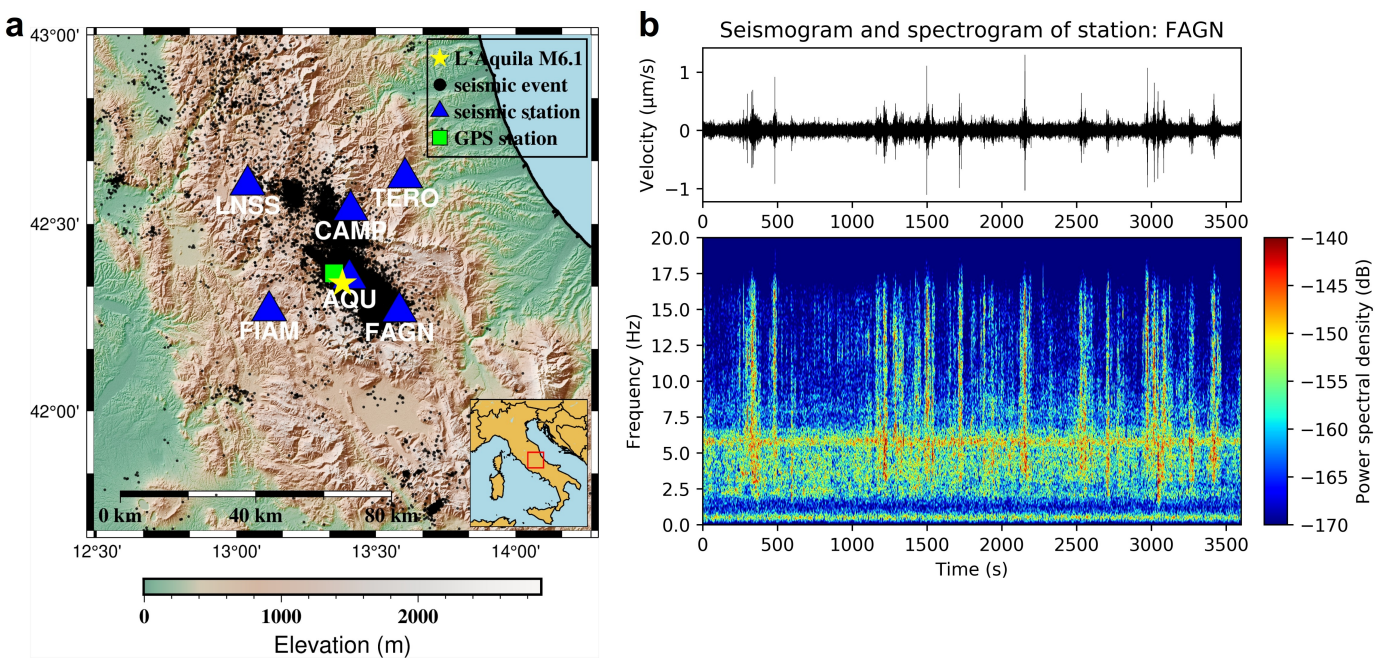

Figure 1. (a) Location of the 2009 L'Aquila earthquake and the nearby permanent seismic array. Yellow star indicates the epicenter of the mainshock. Blue triangles represent the seismic stations. Green square denotes the GPS (Global Positioning System) station. Black dots show the locations of earthquakes including the foreshocks and aftershocks of the 2009 L'Aquila earthquake from 2008-2010 in this region (seismic catalog from INGV). Red rectangular in the bottom-right inserted regional map highlights the current study area. (b) Hour-long example of vertical ground velocity and corresponding spectrogram recorded at the station FAGN. The records start at 2009-04-05 10:00:00 (UTC).

orders of magnitude lower than the average signal amplitudes) to allow a continuous analysis of seismic data and eliminate data anomalies. We have tested that this random noise is not affecting our subsequent analysis. Spectral analysis of the continuous data shows the dominant frequency range of the local earthquakes is around $0.5-18 \mathrm{~Hz}$ (Figure 1b), while below $0.5 \mathrm{~Hz}$, micro-seismic noise dominates. We thus focus on the frequency range of $0.5-18 \mathrm{~Hz}$ to reduce the effects of ambient noise and also influence of regional or remote earthquakes.

\section{Decomposition of the Wavefield and Features Extraction}

\subsection{Covariance Matrix Analysis of Continuous Seismic Data}

We define a set of features relevant for characterizing the propagation of seismic waves beneath the seismic array, over a broad frequency range $(0.5-18 \mathrm{~Hz})$. Following Seydoux et al. (2016a), we extract these features from the factorization of the covariance matrix of continuous array seismic data. Such analyses were successfully used for detecting and classifying seismovolcanic tremors (Soubestre et al., 2018), teleseismic earthquakes (Seydoux et al., 2016a), and for analyzing ambient noise wavefield (Seydoux et al., 2016b).

The covariance matrix is built from the time average of the Fourier cross-spectra matrices calculated over a set of half-overlapping sub-windows (Seydoux et al., 2016a, and Figure 2a). Two types of time window are involved in the calculation of covariance matrix. A short one (sub-window, W1) where the Fourier cross-spectra matrix is calculated, and a longer one (averaging-window, W2) used to average the cross-spectra matrices, which in turn defines the covariance matrix of a particular time scale (Figure 2a). We here use a backward-looking approach to time stamp the results: the end time of each averaging time window (W2) is assigned as the time stamp associated with the covari- 
ance matrix of the time window, hence the obtained results are causal. The size of W1 depends on the size of seismic array and the frequency range of interest (Seydoux et al., 2016a). In this study, we use a W1 of 80 seconds to ensure the slowest waves to fully travel the aperture of the seismic array.

The size of W2 is crucial to define the time resolution of our analysis. We here aim at classifying long-lasting patterns in the seismic signals, and thus we average the covariance matrix over 60 days and shift W2 by one day. The use of long averaging window would probably increase the influence of external wavefield properties originated outside the seismic array. However, as we perform our analysis at relatively high frequencies $(0.5-18 \mathrm{~Hz})$, the analysis inherently focuses on a local area (i.e. inside the array) due to the attenuation of high-frequency waves generated from distant sources. Because we want to analyze seismic sources seen by the ensemble of seismic stations, we apply spectral whitening to the daily seismograms before computing the covariance matrix (Seydoux et al., 2016a, 2016b). In this way, the spectral energy is not taken into account and the analysis mostly relies on the phase coherence between the seismic stations, thus cancelling non-propagative signals (e.g. local noise, traffic, wind).

\subsection{Wavefield Features}

From the eigendecomposition of the covariance matrix, the eigenvalues $\lambda(f, t)$ and corresponding eigenvectors $\mathbf{v}(f, t)$ are obtained for each frequency $f$ and time $t$ (Figure 2a). Note that the covariance matrix is inherently Hermitian and positive semi-definite; the matrix is therefore always diagonalizable and the eigenvalues are positive and real. From the eigenvalues, we define four features: (1) the Shannon entropy, (2) the coherency, (3) the eigenvalue variance, and (4) the first eigenvalue.

1. The Shannon entropy, initially developed in the frame of information theory (Shannon, 1948) and applied to the case of discrete operators by Von Neumann (1986), provides a measurement of the quantity of information present in a multivariate dataset. If we consider the normalized covariance matrix eigenvalues $p_{i}(f, t)=\lambda_{i}(f, t) / \sum_{i=1}^{N} \lambda_{i}(f, t)$ such as $\sum_{i=1}^{N} p_{i}(f, t)=1$ (where $N$ is the total number of stations in the array and $p_{i}$ represents the normalized $i$-th eigenvalue of the covariance matrix at a given time and frequency), we can consider each normalized eigenvalue $\left(p_{i}\right)$ to represent the probability of each source (identified by each corresponding eigenvector) to be observed in the studied time period. The Shannon entropy $\sigma_{e}$ is then defined as:

$$
\sigma_{e}(f, t)=-\sum_{i=1}^{N} p_{i}(f, t) \ln \left(p_{i}(f, t)\right)
$$

Following Shannon (1948), the higher the entropy, the more chaotic the wavefield and the lower the wavefield spatial coherence. A coherent wavefield generated by only one source or many co-located sources in the analyzed time window is likely to be spanned by a single dominating eigenvalue (Figure $2 \mathrm{~b}$ ). Therefore, low values of the entropy will be observed when the wavefield is dominated by the coherent sources localized in space.

2. The coherency function, commonly used in exploration geophysics (Gersztenkorn \& Marfurt, 1999), is defined as the ratio between dominating wavefield component (first eigenvalue) and the full wavefield (sum of all eigenvalues), and reports the wavefield coherence $\sigma_{c}$ :

$$
\sigma_{c}(f, t)=\frac{\lambda_{1}(f, t)}{\sum_{i=1}^{N} \lambda_{i}(f, t)} .
$$


3. To estimate the flatness of covariance matrix eigenvalues distribution, we define the eigenvalue variance $\sigma_{v}$ as:

$$
\sigma_{v}(f, t)=\frac{\sum_{i=1}^{N}\left(\lambda_{i}(f, t)-\mu\right)^{2}}{N},
$$

where $\mu=\sum_{i=1}^{N} \lambda_{i}(f, t) / N$ is the mean eigenvalue at a given time and frequency. The eigenvalue variance is related to both wavefield coherence and source energy (Figures 2b2d). For example, for one dominating source in the studied time window (W2), the corresponding eigenvalue variance will be large and the wavefield is coherent as well.

4. Finally, we use the first eigenvalue $\sigma_{f}$ :

$$
\sigma_{f}(f, t)=\lambda_{1}(f, t) .
$$

Theoretically this value defines the coherence of a single source over the time window W2. As it is resulting from phase multiplication, this value can be affected by noise, for example biasing the estimation of the phase correlation. There is thus an imprint of the frequency dependent signal-to-noise level in this measure. For example, stronger source and/or a large number of co-located coherent sources in the studied time window (W2) will result in larger phase correlations (because of higher signal-to-noise ratio after averaging) and thus lead to a larger eigenvalue. Therefore, the first eigenvalue provides a measurement of the strength of the dominating source in the wavefield.

These four features are obtained at each time step (1 day) and frequency (from 0.5 to $18 \mathrm{~Hz}$ ). We thus have a time-frequency representation of the wavefield (Figure 2a), which can be used to track its evolution. As mentioned above, the features contain insights about the wavefield spatio-temporal properties, and thus provide insights on the seismic signals generated inside the array. Since the wavefield features are calculated using a long window (60 days), many seismic sources can exist in the same time window of analysis. Among the different potential scenarios, we can distinguish the following extreme cases.

If many seismic sources occur in a small region with respect to the wavelength and the array aperture (e.g. an earthquake swarm or co-located sources), the average covariance matrix will exhibit a dominant eigenvalue while the other eigenvalues will be small (scenario illustrated in Figure 2b), giving small values for the entropy and high values for the coherency, eigenvalue variance and first eigenvalue.

If many independent seismic sources are acting in the same time window (W2) and scattered in a vast area with respect to the array aperture, the eigenvalue distribution will follow a steadily decaying distribution (scenario illustrated in Figure 2c) specific to the array geometry, the structure of the underlying medium and the duration of the averaging window W2 (Seydoux et al., 2016a). In this situation, the entropy and first eigenvalue will be high and the coherency and eigenvalue variance will be small, indicating an incoherent ensemble wavefield with many incoherent seismic sources in the analyzed time scale (W2).

Finally, if the records only contain electronic noise or spatially distributed incoherent perturbations (e.g. rain, wind, road traffic etc.), the covariance matrix eigenvalues will be approximately equal and small (scenario illustrated in Figure 2d) depending on the estimation parameters (Menon et al., 2014). In this situation, the entropy will be high and the coherency, eigenvalue variance and first eigenvalue will be small, indicating an incoherent ensemble wavefield with no sources in the analyzed time scale (W2).

In summary, the defined wavefield features permit to discern the behavior of the wavefield over different frequencies and as a function of time. We use these features to 
Averaging time window (W2)

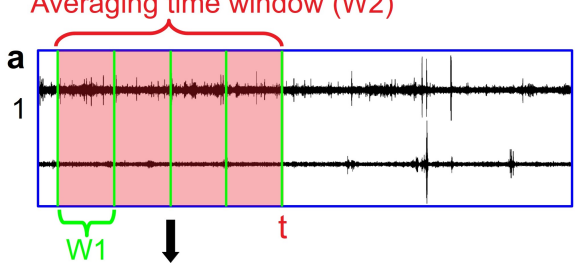

2
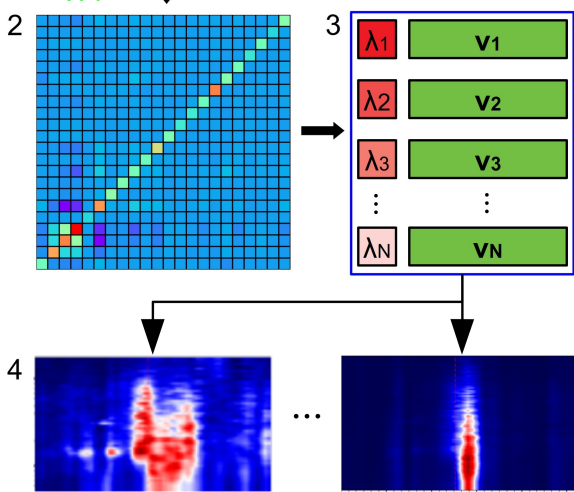
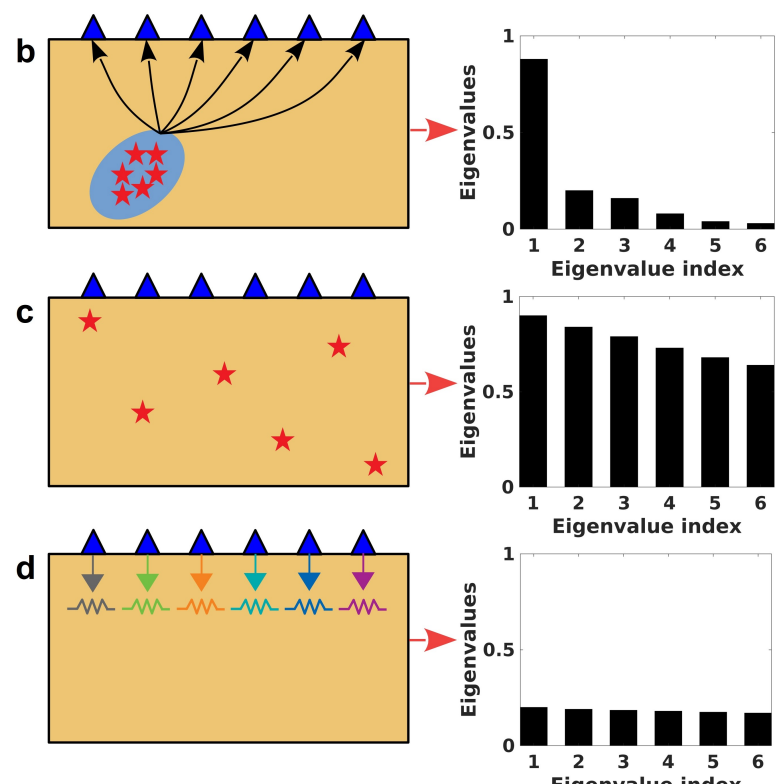

Figure 2. (a) Workflow of wavefield features extraction and analysis, which includes: 1. seismic data processing (e.g. filtering etc.) and time window determination, 2. covariance matrix calculation, 3. eigendecomposition of covariance matrix, and 4. wavefield feature extraction. Right panel shows three representative scenarios of source distribution and the corresponding eigenvalue distribution of covariance matrix in the time window of analysis, which are (b) many co-located seismic sources, (c) many independent (spatially scattered) seismic sources, and (d) electronic or local non-seismic sources. Blue triangles indicate seismic stations and red stars indicate seismic sources.

track the evolution of the wavefield during the seismic cycle (short term in this case, 3 years), and to assess if seismic signals contain information about the evolution of the fault state.

\section{Feature Analysis and Clustering}

\subsection{Feature Relationship and Sensitivity Analysis}

The extracted wavefield features over the full dataset are shown in Figure 3 as a function of time and frequency over the 3 years centered on the L'Aquila earthquake. In particular, the coherency and entropy features (Figures 3a and 3b) are increasing and dropping respectively before the mainshock, suggesting the activation of localized sources in the 3 months before the mainshock at 1-10 Hz. After the strike of the mainshock, during the aftershock sequence, the frequency content of the coherent wavefield moves to a lower frequency range (below $5 \mathrm{~Hz}$ ). Yet, depending on the ratio between the wavelength of the seismic wavefield and the seismic array aperture, multiple sources distributed in space are likely to induce a low coherence value (as depicted in Figure 2c). As observed in the wavefield features (Figures 3a and 3b), at high frequencies, the coherence almost vanishes, whereas at lower frequencies (below $5 \mathrm{~Hz}$ ), a high coherence is still observed (due to larger wavelengths). This is in agreement with the spread of aftershocks near the rupture zone due to the stress redistribution after the mainshock. The eigenvalue variance and first eigenvalue features (Figures $3 \mathrm{c}$ and $3 \mathrm{~d}$ ) indicate that the fault is most active during the aftershock periods. In addition, the eigenvalue variance tends to increase 

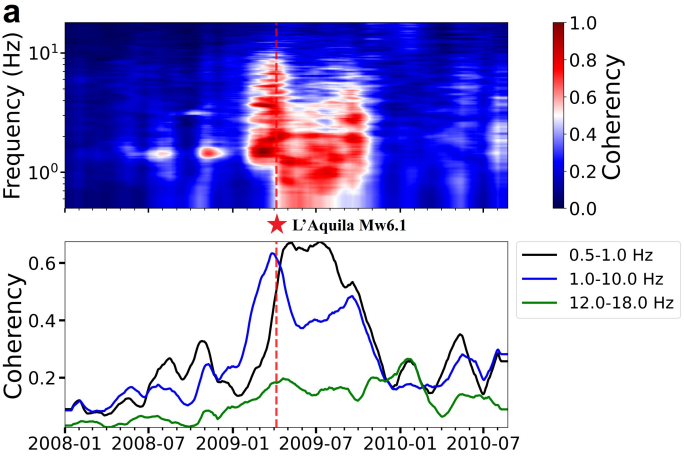

C
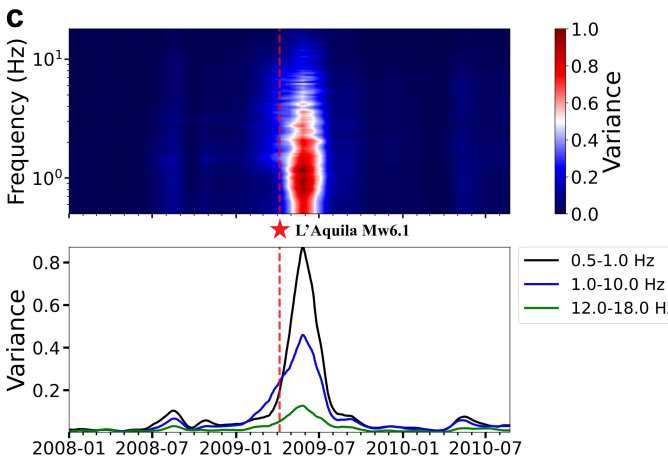
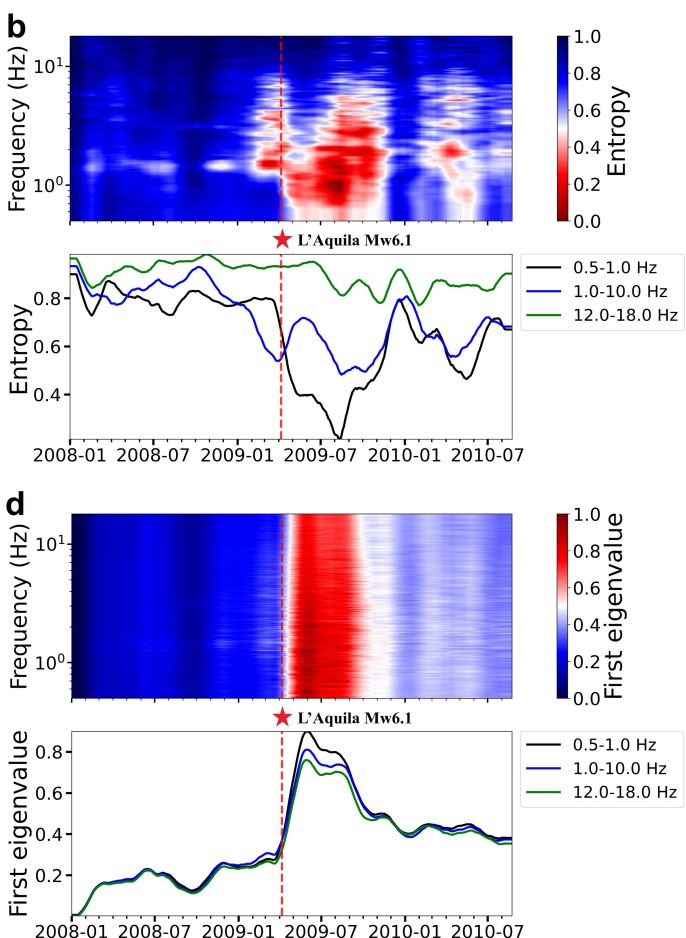

Figure 3. The extracted wavefield features using an averaging window of 60 days. For each sub-figure, the top panel shows the feature with respect to time and frequency (frequency axis in log scale, ranges from 0.5 to $18 \mathrm{~Hz}$ ), and the bottom panel shows the features averaged in three different frequency bands. The horizontal axis shows time (ranges from 2008-01-01 to 2010-0823). The red dashed line and star highlight the origin time of the 2009 L'Aquila earthquake. (a) Coherency; (b) Entropy; (c) Eigenvalue variance; (d) First eigenvalue.

as the mainshock is approaching, especially in the frequency range of $1-10 \mathrm{~Hz}$, suggesting an activation of relatively strong sources in the area (Figure 3c). The overall timefrequency evolution of the wavefield features in the studied region visually suggests that different physical processes are acting during the pre- and post-seismic stages.

To quantitatively asses if the observed features can isolate different stages of the seismic cycle (e.g. pre- and post-seismic) we apply an unsupervised class-membership identification (clustering). Our approach is similar to the clustering of laboratory data of Bolton et al. (2019). Our scope is to naturally separate periods with potential different physical processes in the fault region, solely from data. We thus avoid any explicit physical modeling (e.g. location of events, magnitude estimation) and time constrain (e.g. before and after the earthquake), and learn relevant characteristics with implicit models from the data itself.

Visually, some of the proposed features (e.g. entropy and coherency, Figure 3) show some similarities, and will be likely redundant in the identification of classes. To quantify any redundancy in our dataset, we analyze the relationship between wavefield features and select the uncorrelated ones (i.e. features that are independent and responsible for different source properties) for clustering.

To that scope, we calculate the correlation coefficients between different features in different frequency ranges. Results of this analysis are reported in Figure 4. The entropy and coherency, which provide an estimate of the wavefield coherence, are well cor- 

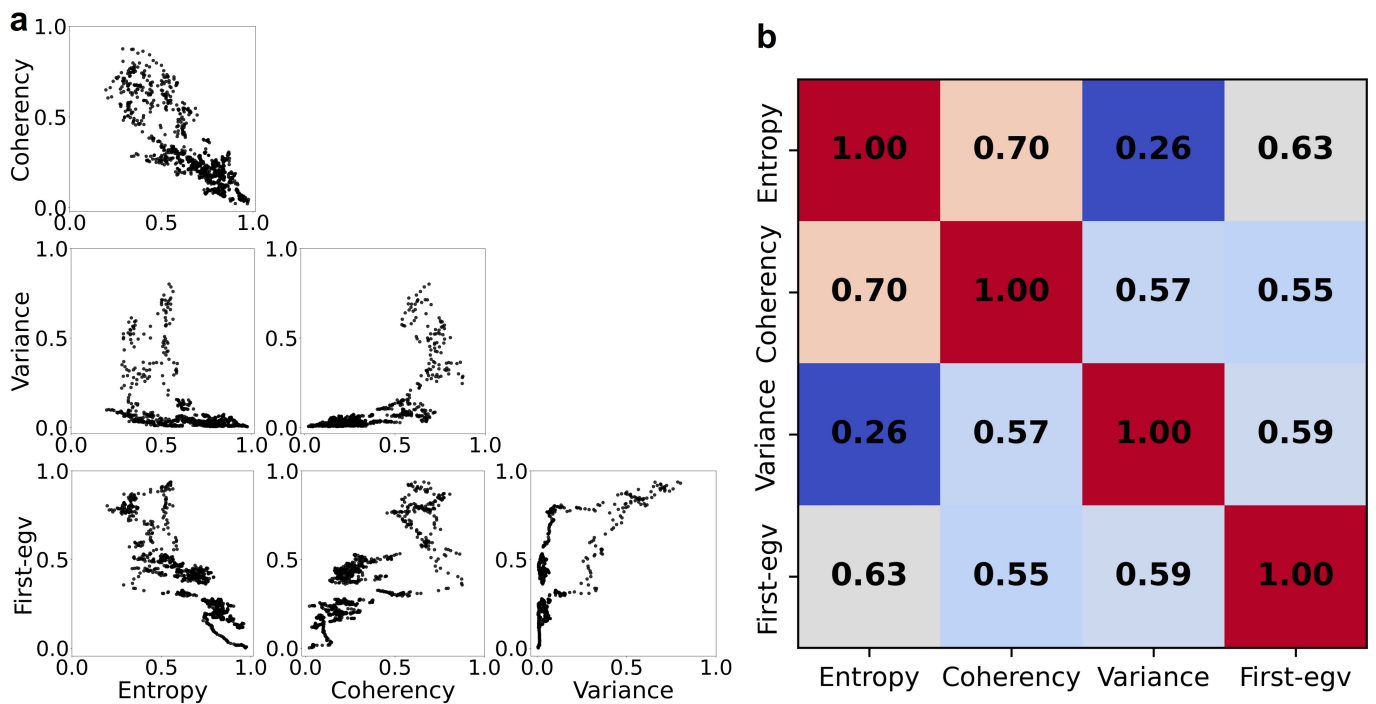

Figure 4. Correlation analysis between different features. (a) Cross-plot of different features at frequency band: $2-2.1 \mathrm{~Hz}$ (corresponds to the lower triangular part of the correlation coefficient matrix in (b)). (b) Average correlation coefficients between different features over the full frequency range $(0.5-18 \mathrm{~Hz})$. Correlation coefficients are first calculated based on the average features of $0.1 \mathrm{~Hz}$ frequency bins, and then are averaged to obtain the final correlation coefficients over the whole frequency band.

related with each other over a broad frequency range $(0.5-18 \mathrm{~Hz})$ with an average correlation coefficient of 0.7 . The eigenvalue variance shows an average correlation coefficient of 0.57 and 0.59 with the coherency and first eigenvalue respectively, which indicates it contains information about the wavefield coherence and the source energy at the same time.

\subsection{Cluster Analysis}

According to the sensitivity analysis of all features (Section 4.1), the coherency, eigenvalue variance and first eigenvalue are poorly correlated (Figure 4) indicating a sensitivity to different properties of the wavefield (Figures 2 and 3). These three features are thus selected for the unsupervised analysis. For each time window, the number of frequency points is large (2800 points), therefore defining a very large feature space of 3 x 2800 dimensions. In order to reduce the dimension of the feature space, we focus on the sensitive frequency range $(0.5-10 \mathrm{~Hz})$ and average each feature in frequency bins of $0.1 \mathrm{~Hz}$ from 0.5 to $10 \mathrm{~Hz}$. We end up with 95 frequency bins for each of the three features. In addition, we linearly normalize the feature magnitude in the interval $[0,1]$ with the feature maximum over all the frequencies in order to balance the information provided by each feature (e.g. Bolton et al., 2019). In this way, the relative amplitude of the features in different frequency bins is kept. Finally, the three normalized features are combined together, forming a feature space of 285 dimensions $(3 \times 95)$ for cluster analysis.

We extract 966 samples (time segments of W2) in total over the dataset for clustering analysis. Clusters found in seismic data are likely to be unbalanced, because different physical processes may occur at different timescales (e.g. seismic data are mostly composed by noise). Yet, many clustering approaches are essentially based on the cluster size balance in order to evaluate the clustering quality (for instance K-Means). More 
generally, class imbalance is a general issue in clustering, and only few algorithms allow to overcome this problem. Hierarchical clustering (Maimon \& Rokach, 2005) is recognized as one of the most powerful approach to cluster unevenly distributed class of data. This is done by building a hierarchy of nested clusters by successively merging or splitting data samples based on any pairwise distance between the data points. In this study, we use an agglomerative strategy which treats each data sample as a cluster and successively merges the two clusters with the smallest distance until all clusters are gathered by a root cluster (Pedregosa et al., 2011). We use L1 distance to measure the distances between data samples.

The hierarchy of our clustering can be represented by a dendrogram, which indicates the distance and splitting between clusters (Figure 5a). We then use a silhouette analysis (Rousseeuw, 1987) to determine the optimal number of clusters (Figures 5b and $5 c$ ). The silhouette score is a measure of the average distance between a sample in one cluster to the samples in the neighboring clusters and thus provides a way to assess cluster separation. It is calculated from the normalized difference between the mean nearest inter-cluster distance and the mean intra-cluster distance. Therefore, a large average silhouette score generally indicates large separating distances between the resulting clusters, and hence better clustering results. We vary the number of clusters between 3 to 15 , and found that 6 clusters allow to achieve the best separation (Figures $5 \mathrm{~b}$ and $5 \mathrm{c}$ ).

\subsection{Clustering Results}

Because the dimension of the feature space is large, we propose to visualize the clustering results from the two main principal features components. We extract these components with principal component analysis (PCA) as shown in Figure 6. PCA projects data from the original feature space into a principal component (PC) space. Each PC is a linear combination of all the original features, scaled by a corresponding correlation coefficient. PCA also allows to observe the data variance explained by each component. In our case, we see that the first three PCs (PC1-PC3) respectively explain about $80 \%$, $10 \%$ and $6 \%$ of the total data variance, while all other PCs account for less than $1 \%$ of the total data variance each (Figure 6a). Since the first two PCs account for almost $90 \%$ of the data variance together, we can thus effectively represent and visualize our data in a $2 \mathrm{D} \mathrm{PC}$ space.

We use PCA to identify the most relevant wavefield features and frequency ranges to each PC by looking at the linear combination coefficients of the original features, which is useful to interpret the clustering results in a more physical way (Figure 6b). The PCA results indicate that the first $\mathrm{PC}$ is highly correlated with the first eigenvalue (Figure $6 \mathrm{~b}$ ), while the second PC is highly related to the wavefield coherence (Figure 6b).

The clustering results are presented in the space formed by the first two principal components in Figure 7. Six clusters are presented along with other independent measurements, i.e. GPS displacement and seismic catalog (Figure 7c). As shown in Figure $7 \mathrm{a}$, the six clusters are well separated in the PC space indicating there are clear and well recognizable patterns in the continuous seismic wavefield. The distribution of different clusters in the original feature space also demonstrates the clustering results are a natural partition according to the wavefield property variations (Figures 8 and 9). The temporal evolution of the clustered data points is shown in the PC space (Figure $7 \mathrm{~b}$ ) and corresponding to each measurement (i.e., PC1-PC3, GPS and seismic catalog, Figure 7c). In Figure 7c, the different PCs, GPS measurements and seismic catalog are color-coded according to the identified clusters to better observe differences among the different clusters.

Before discussing the properties for each cluster, it is worth to remind that the features are extracted from 60 days of data, and each point in Figure 7 is at the end of the time window. Thus, each point has seen data for the preceding 60 days (see Figure 7c), 

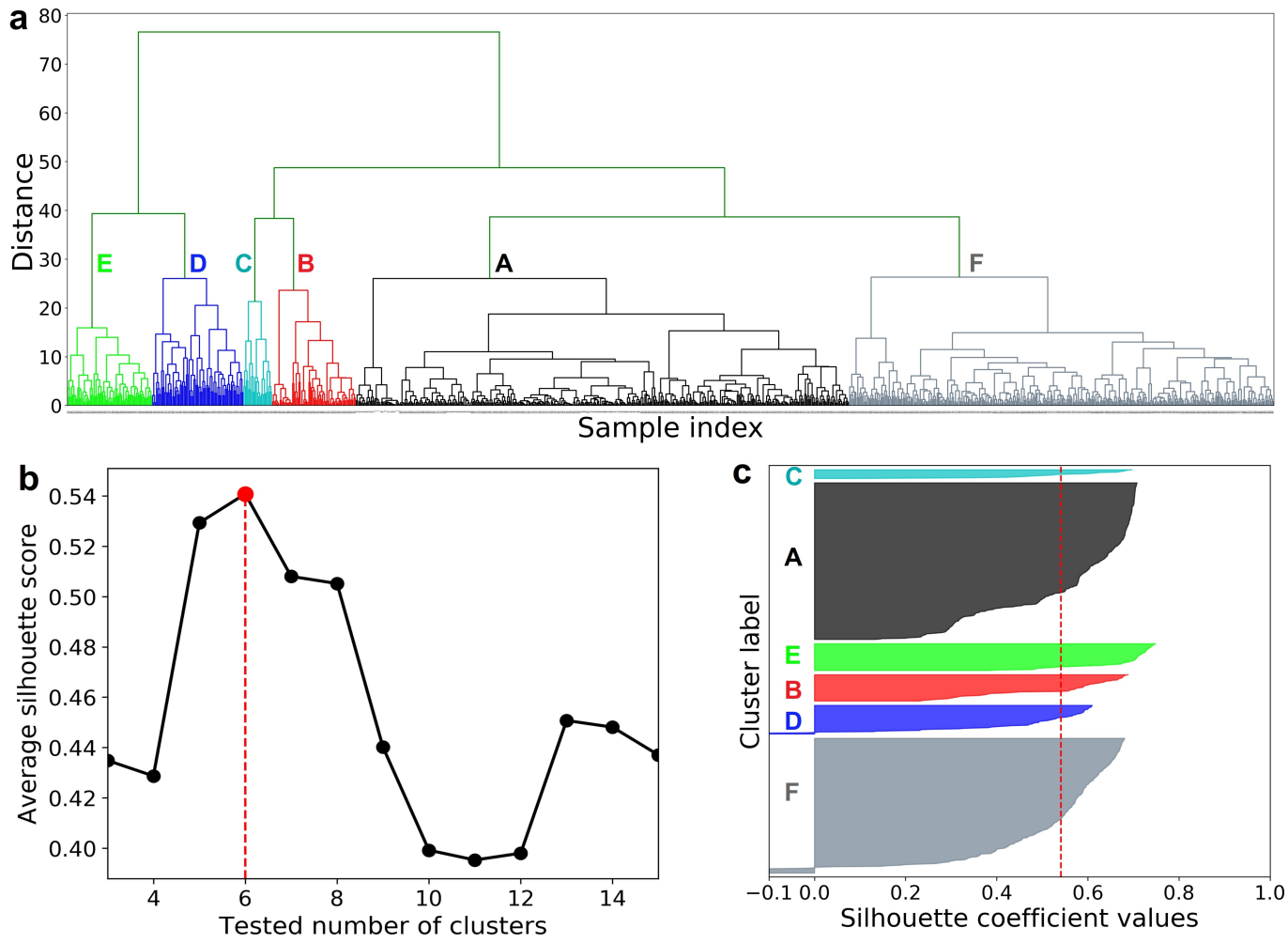

Figure 5. (a) Dendrogram of hierarchical clustering. Different clusters are marked by different colors and annotated using the cluster labels: A-F. The color-code and label of different clusters are consistent with that in Figure 7. The sample index correspond to the date index. (b) Variation of average silhouette score with the number of clusters. Red dashed line indicates that when the number of clusters is 6 , the silhouette score reaches to a maximum of about 0.54. (c) Silhouette scores of the data points in each cluster when the number of cluster is 6 . Different colors correspond to different clusters. Red dashed line shows the average silhouette score. Most data points in the six clusters have a silhouette score larger than the average score, which indicates a favorable clustering result. 

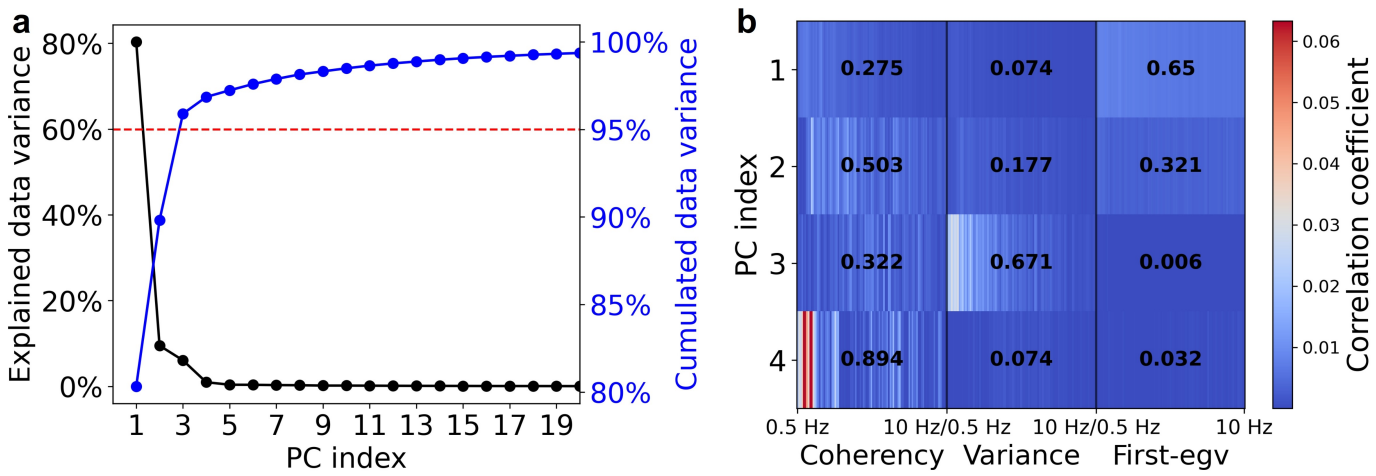

Figure 6. PCA of all the input features. (a) Black line shows the explained data variance (in percentage) of the first 20 principal components (correspond to the left axis). Blue line shows the cumulative explained data variance for the number of principal components used (correspond to the right axis). Red dashed line highlights $95 \%$ cumulative percentage. (b) The correlation coefficients between the first four principal components and the features in different frequency bins. The number marked on each section shows the cumulative correlation coefficient over the whole adopted frequency range $(0.5-10 \mathrm{~Hz})$ for the coherency, eigenvalue variance and first eigenvalue feature, respectively.

and for example, cluster $\mathrm{C}$ contains a mixture of signals from times prior and after the mainshock.

Cluster A identified with a low wavefield coherency (Figures 3a, 3b, and 9) and small first eigenvalues (Figures 3d and 9), corresponds to a quiet period (low seismicity). Cluster B exhibits increased wavefield coherency (especially in the frequency band of 1-10 $\mathrm{Hz}$, see in Figures 3a, 3b, and 9), eigenvalue variance (Figure 3c and 9), and first eigenvalues (Figures 3d and 9). It corresponds to the increment of seismic activity prior the 2009 L'Aquila earthquake. During this period, the earthquake rate increased in this region (Sugan et al., 2014; Vuan et al., 2018) and the earthquakes also tend to localize around the fault (Figures $7 \mathrm{c}$ and 9 ).

Clusters $\mathrm{C}$ is likely resolving the last period before the main event, but is also affected by the mainshock and some aftershocks. It is showing clear differences respect to $\mathrm{A}$ and $\mathrm{B}$, in particular an increment of first eigenvalue and a reduction of coherency at 1-10 Hz (Figures 3a, 3d, 7c and 9). The group D, which shows strong wavefield coherency in the low frequency range $(0.5-1 \mathrm{~Hz})$ and large first eigenvalues (Figures 3a, 3d, and 7c), corresponds to a short period of aftershock sequences immediately after the 2009 L'Aquila earthquake.

Compared with cluster D, cluster E shows increasing wavefield coherency (at 1-10 $\mathrm{Hz}$ ) and decreasing first eigenvalues (Figures 3, 7c and 9). It is worth to note that although both clusters $\mathrm{D}$ and $\mathrm{E}$ fall into aftershock sequences, they exhibit distinct coherency variations in different frequency ranges (0.5-1 and 1-10 Hz, see in Figures 3 and 9). Moreover, there is a jump in $\mathrm{PC} 2$ from cluster D to E (Figure 7c). According to the PCA analysis (figure 6), PC2 is mainly related to wavefield coherency. Therefore, the jump in PC2 from cluster $\mathrm{D}$ to $\mathrm{E}$ is mainly due to a change in the wavefield coherency, which can be confirmed in the extracted coherency feature (Figures 3 and 9). Compared with cluster D, the wavefield coherency of cluster E increases at 1-10 Hz. This behavior can be interpreted (see in Figure 2) as an activation of localized seismic sources of low magnitudes (especially the event cluster in $30 \mathrm{~km}$ away to the main event, Figure 9). These observations suggest an evolution of the aftershock behavior. During the period of clus- 
a

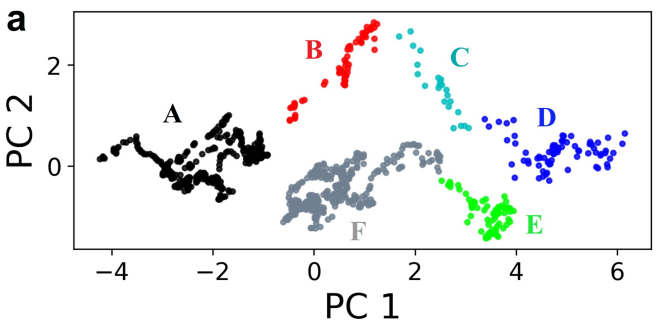

Cluster A

Cluster D

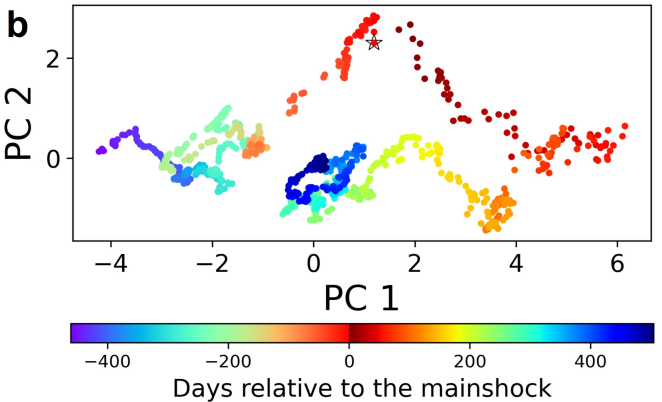

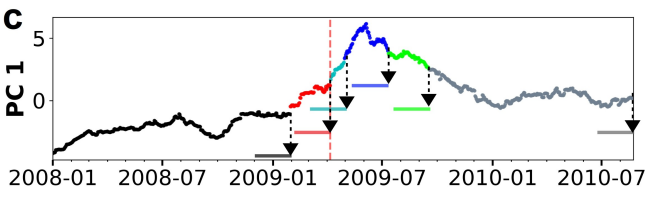

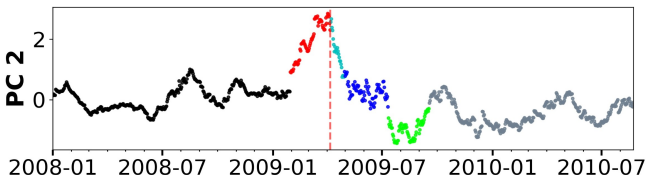

2008-01 2008-07 2009-01 2009-07 2010-01 2010-07
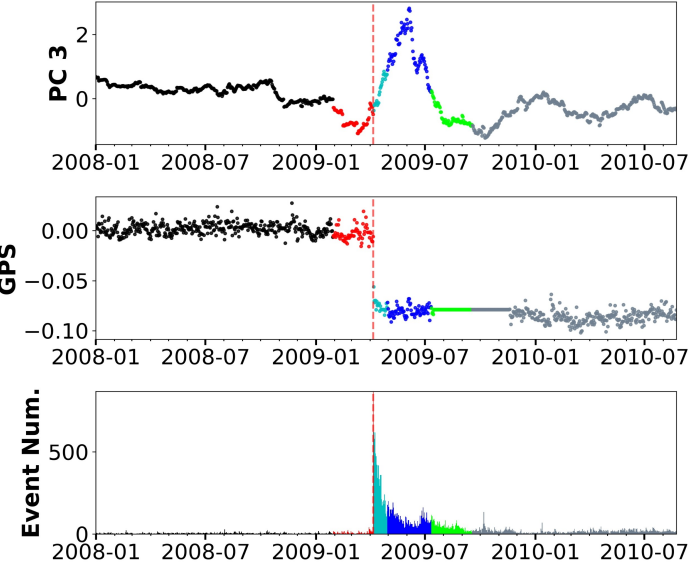

Figure 7. Clustering results shown in the 2D PC space with horizontal axis showing the first PC and vertical axis showing the second PC. Six clusters are color-coded and marked with labels A to F (consistent with Figure 5). (b) Temporal variation of the clustered points in the 2D PC space. The data points are color-coded according to the days relative to the mainshock as indicated by the colorbar at the bottom. The star highlights the day when the 2009 L'Aquila earthquake occurred. (c) Temporal variation of the principal components, GPS measurements and number of seismic events per day. The red dashed lines exhibits the origin time of the 2009 L'Aquila earthquake. The first to third rows show the variation of the first three PCs with time. The fourth row shows the ground displacements in the vertical direction measured by a GPS station in L'Aquila (location shown in Figure 1a). The fifth row shows the detected number of seismic events per day in the INGV catalog. The different measurements are color-coded according to the corresponding cluster. The time window (60 days) for extracting wavefield features at the last data sample in each cluster is highlighted by the black arrow and the corresponding color-coded bar in the top panel.

ter $\mathrm{E}$, the earthquake rate is much lower than the previous aftershock stages $(\mathrm{C}$ and $\mathrm{D})$ and localized swarm-like seismicity of low magnitudes emerges (Figures $7 \mathrm{c}$ and 9).

The last cluster (F) shows low wavefield coherency and steady decreasing first eigenvalues (Figures 3 and 7). During this period, the aftershocks sequence reduces and the earthquake rate in the region starts to recover to a background level (Figures 7c and 9). As shown in the dendrogram (Figure 5a) and in Figure 7a, the A and F clusters are close to each other and belong to the same root cluster. Compared to the other clusters which are more seismically active, they correspond to quieter periods. 

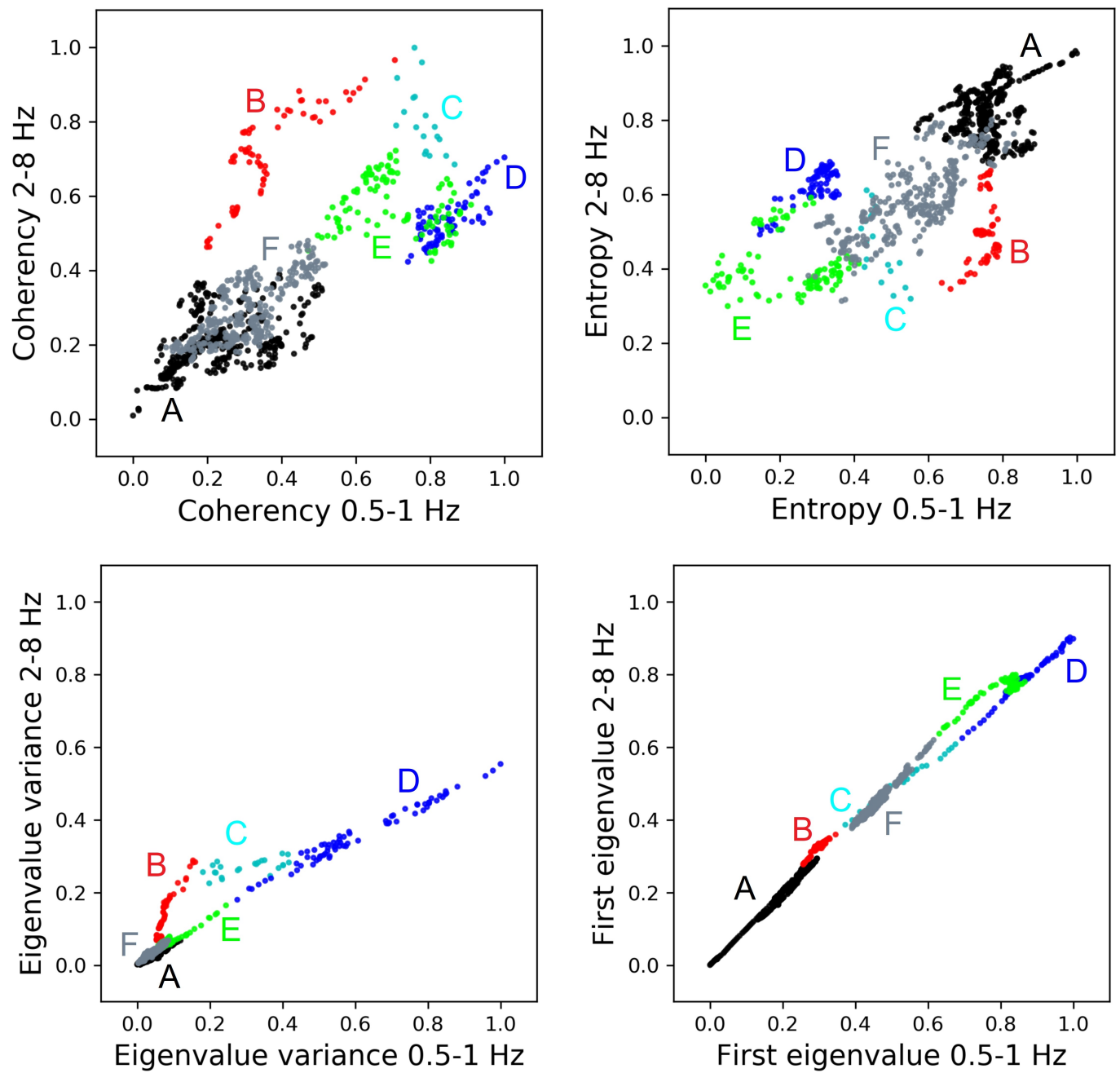

Figure 8. The distribution of the identified clusters in the wavefield feature space. The wavefield features are extracted at each frequency point from $0.5-18 \mathrm{~Hz}$. Here for better visualizing the clustering results in a $2 \mathrm{D}$ feature space, the features are averaged and shown in two frequency bands, which are (1) low frequency band: $0.5-1 \mathrm{~Hz}$ and (2) higher frequency band: $2-8 \mathrm{~Hz}$. 

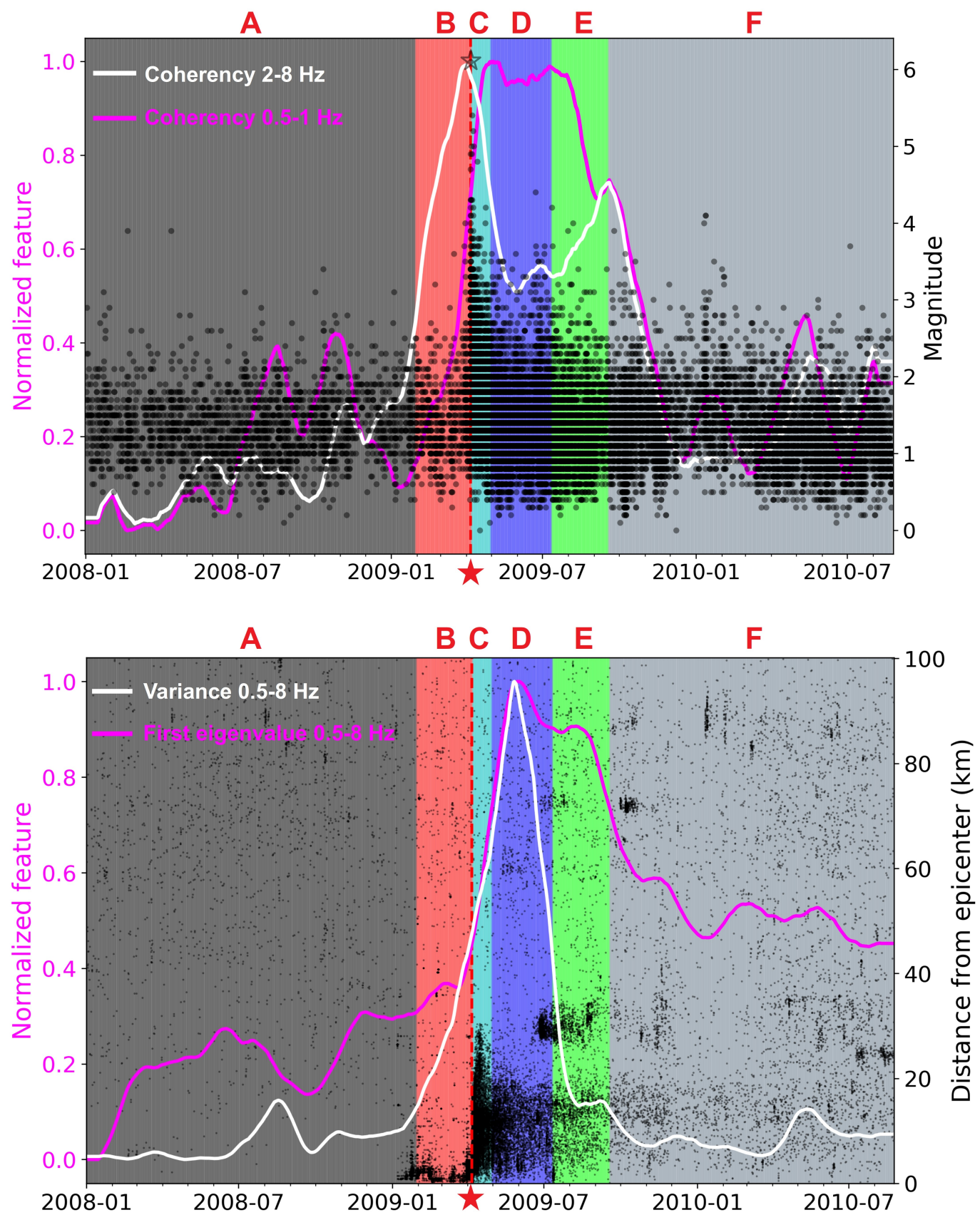

Figure 9. Clustering results with different colors representing different clusters (clusters: A-F). Seismic events from INGV catalog and the wavefield features are also displayed for comparison. The origin time of the 2009 L'Aquila earthquake is marked by the red star and red dash line. In the top panel, clustering results are shown together with the average coherency feature in different frequency bands (correspond to the left axis) and local magnitudes of seismic events (correspond the right axis). In the bottom panel, clustering results are shown together with the average eigenvalue variance feature (left axis), the first eigenvalue feature (left axis) and the event distances from the epicenter of the 2009 L'Aquila earthquake (right axis). 


\section{Discussion}

We show the existence of the time and frequency evolution of wavefield features derived from continuous seismic records, and how the analysis of these features reveals distinct clusters well correlated with different periods of the seismic cycle (Figure 7). As the analysis is performed over long-term scale, the time-frequency evolution reflects the statistical wavefield properties and is related to the evolution in position, size, number and distribution pattern of the seismic sources inside the array (Figure 2). Hence, by analyzing the statistical properties of continuous wavefield, we draw conclusions about physical processes occurring in the fault region without the need of any modeling.

As the used features have physical meanings, they can provide important information about the processes occurring in each cluster. For example, cluster B, which is characterized by increasing coherence and first eigenvalue, suggests the activation of localized sources prior to the main event (see Figures 2, 3 and 7). This behavior agrees with previous studies on L'Aquila earthquake, suggesting the occurrence of localized foreshocks and increased earthquake rate before the mainshock (Sugan et al., 2014). However, differently from previous studies, no explicit modeling is involved in our analysis, and we show how this information emerges naturally from our chosen representation of the continuous seismic wavefield. Clusters D and E show a reduction of the coherency compared to clusters $\mathrm{B}$ and $\mathrm{C}$ especially in the high frequency range $(1-10 \mathrm{~Hz})$. This behavior suggests that seismicity is spread around the fault, as stress is redistributed after the mainshock (Marsan, 2005). Previous study based on earthquake catalog (Marsan et al., 2014) shows that earthquakes preceded by accelerating seismicity rate produce more aftershocks on average and exhibit more spatial spreading aftershock sequences, which agrees with our model free analysis here. A similar phenomenon has also been recently observed for the Ridgecrest earthquake (Trugman et al., 2020; Ross et al., 2019), where the temporal and spatial variations of the earthquake waveform similarity before and after the 2019 Ridgecrest earthquake are compared. Significant reduction of the earthquake similarity in the aftershock sequences (compared to the pre-event seismicity) is observed and interpreted as a result of small scale heterogeneities in the residual stress field initiated by the main event. Their observations of the temporal variation of coherence using earthquake waveforms of catalogued events correspond well with our results derived from continuous seismic data. But again, our observations and analysis are model free and do not require additional seismological dateset such as earthquake catalogs and velocity models.

More complex is the interpretation of cluster C, which partially covers the last preseismic period and a portion of time after the event. This issue comes from the limitation of our methodology to a given timescale. In fact, the use of a long-term window (60 days) with daily step, reduces the possibility of resolving short-lasting clusters and focuses on long-lasting processes. Attempting to reduce the time window will be the subject of future research. However, despite this limitation the method is clearly highlighting different parts of the seismic cycles (including the quiet period, clusters A and F), without the need of modeling.

As in stick-slip rock failure experiments in laboratory (Bolton et al., 2019), our study highlights that fault state can be tracked from continuous seismic data. The ability of unrevealing peculiar patterns in seismic data, extend the laboratory-based idea that continuous data are rich enough to inform us about evolution of physical properties of the fault (e.g. Rouet-Leduc et al., 2017; Bolton et al., 2019). In contrast with the laboratory setting, real data cannot be associated with other boundary conditions (e.g. absolute stress level), and only part of the seismic cycle can be resolved. It is thus unlikely that our features-based approach will permit any kind of machine learning based prediction of the rupture (e.g. Rouet-Leduc et al., 2017). It will however permit to rapidly parse large amount of data and extract peculiar patterns, which can be related to other estimates (e.g. geodetic data) to better characterize different stages of the seismic cy- 
cle. Our features can also be used to regress seismic data into other information (e.g. GPS displacement, Frank et al., 2015) to explore slip rate during aseismic slip episodes.

Finally, in the present study, we defined spatial features for exploring spatially distributed sensors. One of the main advantage is the ability to easily identify propagative signals and to disregard any site-dependent patterns that may bias the analysis (e.g. local noise level). Given the large number of seismic arrays deployed worldwide, the developments of features that account for spatial properties of the wavefield is of great interest and will be in the scope of future studies.

\section{Conclusions}

We analyze the wavefield properties with unsupervised machine learning to directly assess fault state and its temporal evolution from continuous seismic data. Unlike traditional statistical features calculated from single station, we extract frequency-dependent wavefield features from the array covariance matrix analysis, which provide the interpretation of the physical properties of the seismic sources. The array-based wavefield features enable to analyze the overall source properties and its temporal evolution for understanding the fault activities in the study region. Our study shows the value of advanced array processing and machine learning analysis to reveal information embedded in the continuous seismic data. Our study builds a bridge between the laboratory experiments and the real earthquake observations and is a step towards understanding the fault physics. Our future work involves further unraveling hidden signals in continuous seismic data for studying fault physics.

\section{Acknowledgments}

This research received funding from the European Research Council (ERC) under the European Union Horizon 2020 Research and Innovation Programme (grant agreements, 802777-MONIFAULTS). L.S. acknowledges support from the European Research Council under the European Union Horizon 2020 research and innovation program (grant agreement no. 742335, F-IMAGE). Computations were performed using the UGA High-Performance Computing infrastructures CIMENT. The continuous seismic data and seismic catalog used in this study are available at the Istituto Nazionale Geofisica e Vulcanologia (INGV) seismological data center (http://cnt.rm.ingv.it/webservices_and_software/, last accessed March 2020). The GPS data used in this study are available at the Nevada Geodetic Laboratory website (http://geodesy.unr.edu/, last accessed March 2020). Figure 1a was made using the Generic Mapping Tools v.6.0.0 (http://gmt.soest.hawaii.edu/, last accessed March 2020; Wessel et al., 2019). The unsupervised machine learning algorithm used in this study were based on scikit-learn v.0.22.2 (https://scikit-learn . org/, last accessed March 2020). We thank editor Rachel Abercrombie, associate editor Nori Nakata and two anonymous reviewers for their efforts and constructive comments.

\section{References}

Aki, K., \& Richards, P. G. (2002). Quantitative seismology. University Science Books.

Baccheschi, P., De Gori, P., Villani, F., Trippetta, F., \& Chiarabba, C. The preparatory phase of the Mw 6.1 2009 L'Aquila (Italy) normal faulting earthquake traced by foreshock time-lapse tomography. Geology, 48(1), 49-55.

Beroza, G. C., \& Ide, S. (2011). Slow earthquakes and nonvolcanic tremor. Annual Review of Earth and Planetary Sciences, 39, 271-296.

Bolton, D. C., Shokouhi, P., Rouet-Leduc, B., Hulbert, C., Rivière, J., Marone, C., \& Johnson, P. A. (2019). Characterizing acoustic signals and searching for 
precursors during the laboratory seismic cycle using unsupervised machine learning. Seismological Research Letters, 90(3), 1088-1098.

Cara, F., Di Giulio, G., \& Rovelli, A. (2003). A study on seismic noise variations at Colfiorito, central Italy: implications for the use of $\mathrm{H} / \mathrm{V}$ spectral ratios. Geophysical Research Letters, 30(18).

Carniel, R., Jolly, A. D., \& Barbui, L. (2013). Analysis of phreatic events at Ruapehu volcano, New Zealand using a new SOM approach. Journal of Volcanology and Geothermal Research, 254, 69-79.

Chiarabba, C., Amato, A., Anselmi, M., Baccheschi, P., Bianchi, I., Cattaneo, M., ... others (2009). The 2009 L'Aquila (central Italy) MW6.3 earthquake: Main shock and aftershocks. Geophysical Research Letters, 36(18).

Chiarabba, C., Buttinelli, M., Cattaneo, M., \& De Gori, P. (2020). Large earthquakes driven by fluid overpressure: The Apennines normal faulting system case. Tectonics, 39(4), e2019TC006014.

Di Luccio, F., Ventura, G., Di Giovambattista, R., Piscini, A., \& Cinti, F. (2010). Normal faults and thrusts reactivated by deep fluids: The 6 April $2009 \mathrm{Mw}$ 6.3 L'Aquila earthquake, central Italy. Journal of Geophysical Research: Solid Earth, 115(B6).

Di Stefano, R., Chiarabba, C., Chiaraluce, L., Cocco, M., De Gori, P., Piccinini, D., \& Valoroso, L. (2011). Fault zone properties affecting the rupture evolution of the 2009 (Mw 6.1) L'Aquila earthquake (central Italy): Insights from seismic tomography. Geophysical Research Letters, 38(10).

Esposito, A. M., Giudicepietro, F., D’Auria, L., Scarpetta, S., Martini, M. G., Coltelli, M., \& Marinaro, M. (2008). Unsupervised neural analysis of verylong-period events at Stromboli volcano using the self-organizing maps. Bulletin of the Seismological Society of America, 98(5), 2449-2459.

Frank, W. B., Radiguet, M., Rousset, B., Shapiro, N. M., Husker, A. L., Kostoglodov, V., ... Campillo, M. (2015). Uncovering the geodetic signature of silent slip through repeating earthquakes. Geophysical Research Letters, 42(8), 2774-2779.

Gersztenkorn, A., \& Marfurt, K. J. (1999). Eigenstructure-based coherence computations as an aid to 3-D structural and stratigraphic mapping. Geophysics, 64(5), 1468-1479.

Gutenberg, B., \& Richter, C. F. (1956). Magnitude and energy of earthquakes. Annali di Geofisica, 9(1), 1-15.

Herrmann, R. B., Malagnini, L., \& Munafò, I. (2011). Regional Moment Tensors of the 2009 L'Aquila Earthquake Sequence. Bulletin of the Seismological Society of America, $101(3), 975-993$.

Hulbert, C., Rouet-Leduc, B., Johnson, P. A., Ren, C. X., Rivière, J., Bolton, D. C., \& Marone, C. (2019). Similarity of fast and slow earthquakes illuminated by machine learning. Nature Geoscience, 12(1), 69-74.

Ide, S., Beroza, G. C., Shelly, D. R., \& Uchide, T. (2007). A scaling law for slow earthquakes. Nature, $447(7140), 76-79$.

INGV Seismological Data Centre. (2006). Rete Sismica Nazionale (RSN). Istituto Nazionale di Geofisica e Vulcanologia (INGV), Italy. https://doi.org/10.13127/SD/XOFXNH7QFY.

Köhler, A., Ohrnberger, M., \& Scherbaum, F. (2010). Unsupervised pattern recognition in continuous seismic wavefield records using self-organizing maps. Geophysical Journal International, 182(3), 1619-1630.

Langer, H., Falsaperla, S., Masotti, M., Campanini, R., Spampinato, S., \& Messina, A. (2009). Synopsis of supervised and unsupervised pattern classification techniques applied to volcanic tremor data at Mt Etna, Italy. Geophysical Journal International, 178(2), 1132-1144.

Langer, H., Falsaperla, S., Messina, A., Spampinato, S., \& Behncke, B. (2011). Detecting imminent eruptive activity at Mt Etna, Italy, in 2007-2008 through 
pattern classification of volcanic tremor data.

Journal of Volcanology and Geothermal Research, 200(1-2), 1-17.

Lucente, F. P., De Gori, P., Margheriti, L., Piccinini, D., Di Bona, M., Chiarabba, C., \& Agostinetti, N. P. (2010). Temporal variation of seismic velocity and anisotropy before the 2009 MW 6.3 L'Aquila earthquake, Italy. Geology, 38(11), 1015-1018.

Maimon, O., \& Rokach, L. (2005). Data mining and knowledge discovery handbook. Springer.

Marsan, D. (2005). The role of small earthquakes in redistributing crustal elastic stress. Geophysical Journal International, 163(1), 141-151.

Marsan, D., Helmstetter, A., Bouchon, M., \& Dublanchet, P. (2014). Foreshock activity related to enhanced aftershock production. Geophysical Research Letters, 41(19), 6652-6658.

Menon, R., Gerstoft, P., \& Hodgkiss, W. S. (2014). On the apparent attenuation in the spatial coherence estimated from seismic arrays. Journal of Geophysical Research: Solid Earth, $119(4), 3115-3132$.

Pedregosa, F., Varoquaux, G., Gramfort, A., Michel, V., Thirion, B., Grisel, O., ... others (2011). Scikit-learn: Machine learning in Python. Journal of Machine Learning Research, 12, 2825-2830.

Poli, P., Boaga, J., Molinari, I., Cascone, V., \& Boschi, L. (2020). The 2020 coronavirus lockdown and seismic monitoring of anthropic activities in Northern Italy. Scientific Reports, 10(1), 1-8.

Ross, Z. E., Idini, B., Jia, Z., Stephenson, O. L., Zhong, M., Wang, X., ... others (2019). Hierarchical interlocked orthogonal faulting in the 2019 ridgecrest earthquake sequence. Science, 366(6463), 346-351.

Rouet-Leduc, B., Hulbert, C., Lubbers, N., Barros, K., Humphreys, C. J., \& Johnson, P. A. (2017). Machine learning predicts laboratory earthquakes. Geophysical Research Letters, 44 (18), 9276-9282.

Rousseeuw, P. J. (1987). Silhouettes: a graphical aid to the interpretation and validation of cluster analysis. Journal of Computational and Applied Mathematics, 20, 53-65.

Scholz, C. H. (2002). The mechanics of earthquakes and faulting. Cambridge university press.

Seydoux, L., Shapiro, N. M., De Rosny, J., Brenguier, F., \& Landès, M. (2016a). Detecting seismic activity with a covariance matrix analysis of data recorded on seismic arrays. Geophysical Journal International, $204(3), 1430-1442$.

Seydoux, L., Shapiro, N. M., De Rosny, J., \& Landès, M. (2016b). Spatial coherence of the seismic wavefield continuously recorded by the USArray. Geophysical Research Letters, 43(18), 9644-9652.

Shannon, C. E. (1948). A mathematical theory of communication. The Bell System Technical Journal, 27(3), 379-423.

Shreedharan, S., Bolton, D. C., Rivière, J., \& Marone, C. (2020). Preseismic fault creep and elastic wave amplitude precursors scale with lab earthquake magnitude for the continuum of tectonic failure modes. Geophysical Research Letters, 47(8), e2020GL086986.

Soubestre, J., Seydoux, L., Shapiro, N. M., De Rosny, J., Droznin, D., Droznina, S. Y., ... Gordeev, E. (2019). Depth migration of seismovolcanic tremor sources below the Klyuchevskoy volcanic group (Kamchatka) determined from a network-based analysis. Geophysical Research Letters, 46(14), 8018-8030.

Soubestre, J., Shapiro, N. M., Seydoux, L., De Rosny, J., Droznin, D. V., Droznina, S. Y., ... Gordeev, E. I. (2018). Network-based detection and classification of seismovolcanic tremors: Example from the Klyuchevskoy volcanic group in Kamchatka. Journal of Geophysical Research: Solid Earth, 123(1), 564-582.

Sugan, M., Kato, A., Miyake, H., Nakagawa, S., \& Vuan, A. (2014). The preparatory phase of the $2009 \mathrm{Mw} 6.3 \mathrm{~L}$ 'Aquila earthquake by improving the detection 
capability of low-magnitude foreshocks. Geophysical Research Letters, 41(17), 6137-6144.

Trugman, D. T., Ross, Z. E., \& Johnson, P. A. (2020). Imaging stress and faulting complexity through earthquake waveform similarity. Geophysical Research Letters, 47(1), e2019GL085888.

Unglert, K., Radić, V., \& Jellinek, A. M. (2016). Principal component analysis vs. self-organizing maps combined with hierarchical clustering for pattern recognition in volcano seismic spectra. Journal of Volcanology and Geothermal Research, 320, 58-74.

Von Neumann, J. (1986). Papers of John von Neumann on computers and computer theory. The MIT Press, Cambridge, MA.

Vuan, A., Sugan, M., Amati, G., \& Kato, A. (2018). Improving the Detection of Low-Magnitude Seismicity Preceding the Mw 6.3 L'Aquila Earthquake: Development of a Scalable Code Based on the Cross Correlation of Template Earthquakes. Bulletin of the Seismological Society of America, 108(1), 471480.

Wessel, P., Luis, J., Uieda, L., Scharroo, R., Wobbe, F., Smith, W., \& Tian, D. (2019). The generic mapping tools version 6. Geochemistry, Geophysics, Geosystems, 20(11), 5556-5564. 